Research Paper

\title{
CX3CL1 involves in breast cancer metastasizing to the spine via the Src/FAK signaling pathway
}

\author{
Yun Liang\#, Lei Yi\#, Peng Liu, Libo Jiang, Houlei Wang, Annan Hu, Chi Sun, Jian Dong ${ }^{\bowtie}$ \\ Department of Orthopaedic Surgery, Zhongshan Hospital, Fudan University \\ \# Yun Liang and Lei Yi have contributed equally to this study. \\ $\triangle$ Corresponding author: Jian Dong, MD, PhD. Email: Dong.jian@zs-hospital.sh.cn; Address: Department of Orthopeadic Surgery, Zhongshan Hospital, Fudan \\ University, 180 Fenglin Road, Shanghai 200032, People's Republic of China \\ (C) Ivyspring International Publisher. This is an open access article distributed under the terms of the Creative Commons Attribution (CC BY-NC) license \\ (https://creativecommons.org/licenses/by-nc/4.0/). See http://ivyspring.com/terms for full terms and conditions.
}

Received: 2018.04.04; Accepted: 2018.08.04; Published: 2018.09.08

\begin{abstract}
C-X3-C chemokine ligand 1 (CX3CL1) has been shown to be involved in the development of multiple tumors. Our previous study demonstrated that CX3CL1 may be involved in the process of metastasis of various malignant tumors to the spine, including breast cancer, but the molecular mechanism was still unknown. In the present study, we found that the receptor CX3CR 1 was overexpressed in the spinal metastases of breast cancer than in para-tumor tissue. In terms of CX3CL1, it was significantly more expressed in normal spinal cancellous bone than in limbs. However, CX3CR 1 was not expressed at a high level in every breast cancer cell compared with the human mammary epithelial cell line MCF-10A. In addition, CX3CL1 did promote the migration and invasion abilities of MDA-MB-231 cells. However, CX3CL1 has no obvious effect on cell growth. Furthermore, CX3CL1 induced chemotaxis of tumor cells via the Src/FAK signaling pathway. The migration index enhanced by CX3CL1 was dramatically declined using Bosutinib and PF-00562271, which are the inhibitors of Src and FAK signaling pathways, respectively. Therefore, $\mathrm{CX} 3 \mathrm{CLI}$ in spinal cancellous bone attracts $\mathrm{CX} 3 \mathrm{CR} 1$-expressing tumor cells to the spine and enhances their migration and invasion abilities through the Src/FAK signaling pathway.
\end{abstract}

Key words: CX3CL1/CX3CR1; Breast cancer; spinal metastasis; Src/FAK pathway

\section{Introduction}

Spinal metastasis means a malignant tumor originating outside of the bone metastasized to the spine through blood circulation or lymphatic circulation. Approximately $60 \%$ of malignant tumors occur as bone metastasis, of which approximately $50 \%$ have spinal metastasis, such as breast cancer, lung cancer and prostatic cancer $[1,2]$. More than $70 \%$ of end-stage cancer patients had spinal metastasis, as assessed by autopsies [2]. Spinal metastasis may cause serious consequences such as pathologic fracture, severe pain, and spinal cord compression, which seriously affect the patient's quality of life and shorten the patient's lifetime.

However, even in highly malignant primary tumors, therapeutic effects have improved significantly compared with the past, and the prognosis of low or moderate malignant primary tumors is even better, such as a survival rate of breast cancer as high as $90 \%$, which has significantly prolonged survival time [3]. Breast cancer is the most common cancer in women, and with the aging population and dietary changes, the incidence of breast cancer is increasing year by year $[4,5]$. The metastasis of breast cancer is the leading cause of death and thus always a research focus. The most common metastatic target of breast cancer is bone [6], and in clinical settings, we find that spinal metastasis is most frequent $[7,8]$. Therefore, early detection and treatment of breast cancer metastasizing to the spine has represented a challenge for both clinicians and researchers.

CX3CL1, also known as Fractalkine or Neurotactin, has two forms: the membrane-attached form and the shed form [9]. The membrane-attached 
CX3CL1 form mediates firm adhesion of CX3CR1-expressing cells (the only receptor for CX3CL1) to the endothelial cells, and the soluble CX3CL1 form recruits CX3CR1-expressing cells in physiological and pathological processes[9, 10]. Early research found that the CX3CL1/CX3CR1 axis recruited leukocytes, induced angiogenesis, and mediated communication between neurons and glial cells in the central nervous system [9, 11, 12]. However, recent research has demonstrated that the CX3CL1/CX3CR1 axis is involved in the survival, adhesion, and migration of malignant tumor cells, including breast cancer [13, 14]. In our previous research, we first demonstrated that CX3CL1 was associated with the process of spinal metastases from different malignant primary tumors, including breast cancer [15]. Unfortunately, the molecular mechanism of breast cancer metastasizing to the spine still remains poorly understood.

In this study, we confirmed that CX3CR1 was expressed by human breast cancer cell lines and specimens of spinal metastasis. Then, we investigated the role of CX3CL1 in the proliferation and movement of breast cancer cells. Furthermore, the molecular mechanism behind the metastatic process was also investigated.

\section{Materials and Methods}

\section{Patients and tissue specimens}

The patient samples used in this study were all collected from Zhongshan Hospital and included three groups: spinal metastatic breast cancer group, normal spinal cancellous bone group and normal limb cancellous bone group (Table 1). The spinal metastatic breast cancer group consisted of 8 patients who had undergone surgery and whose pathological diagnosis was confirmed by a pathologist. The normal spinal cancellous bone group was 6 patients who received non-tumor spinal surgery. The normal limb cancellous bone group included 6 patients who received joint surgery. The serum was collected before surgery. Informed consent was given and signed by each patient involved in this study before surgery. All procedures were approved by the Ethics Committee of the Zhongshan Hospital and complied with relevant regulation.

\section{Cell lines and cell culture}

The human breast cancer cell lines MCF-7, Bcap-37, MDA-MB-231, MDA-MB-468, and human mammary epithelial cell line MCF-10A were obtained from the Type Culture Collection of the Chinese Academy of Sciences (Shanghai, China). MCF-7, Bcap-37, and MDA-MB-231 were cultivated in Dulbecco's Modified Eagle Medium (DMEM) (Gibco,
MD, USA) containing 10\% fetal bovine serum (FBS, Gibco, MD, USA). MDA-MB-468 cells were cultivated in Leibovitz's L-15 Medium (Gibco, MD, USA) containing $10 \%$ fetal bovine serum at $37^{\circ} \mathrm{C}$ in air without supplementary $\mathrm{CO}_{2}$. MCF-10A cells were cultivated in mammary epithelial cell growth medium without serum.

Table 1. Clinical data of 20 patients

\begin{tabular}{|c|c|}
\hline \multicolumn{2}{|l|}{ Spinal metastatic breast cancer group } \\
\hline No. of cases & 8 \\
\hline Age(years) & 47.25 \\
\hline Gender $(\mathrm{M} / \mathrm{F})$ & $0 / 8$ \\
\hline \multicolumn{2}{|l|}{ Location of lesion } \\
\hline Cervical vertebra & 2 \\
\hline Thoracic vertebra & 5 \\
\hline Lumbar vertebra & 1 \\
\hline \multicolumn{2}{|l|}{ Normal spinal cancellous bone group } \\
\hline No. of cases & 6 \\
\hline Age(years) & 58.3 \\
\hline Gender (M/F) & $2 / 4$ \\
\hline \multicolumn{2}{|l|}{ Diagnosis } \\
\hline Spinal fracture & 2 \\
\hline Spinal degenerative diseases & 4 \\
\hline \multicolumn{2}{|l|}{ Normal limb cancellous bone group } \\
\hline No. of cases & 6 \\
\hline Age(years) & 74.0 \\
\hline Gender $(\mathrm{M} / \mathrm{F})$ & $2 / 4$ \\
\hline \multicolumn{2}{|l|}{ Diagnosis } \\
\hline Femoral neck fracture & 4 \\
\hline Osteonecrosis of femoral head & 1 \\
\hline Knee osteoarthritis & 1 \\
\hline
\end{tabular}

M male, F female

\section{Real-time polymerase chain reaction}

Total RNA of cells and tissue samples was extracted using TRIzol Reagent (Invitrogen, Carlsbad, CA, USA). Then, the RT reagent Kit with gDNA Eraser (Takara bio, Otsu, Japan) was used for reverse transcription. The real-time polymerase chain reaction was performed following the manufacturer's instruction (Takara bio, Otsu, Japan) using a thermocycler (Thermo-ABI 7500, Waltham, MA, USA). The following primers were used in this experiment: $\beta$-actin, forward: 5'-CAACCGCGAGAA GATGACCC-3'; reverse: 5'-GAGGCGTACAGGGAT AGCAC-3'; CX3CR1, forward: 5'-ACATCGTGGTCTT TGGGACT-3'; reverse: 5'-GACACTCTTGGGCTTCTT GC-3'; and CX3CL1, forward: 5'-CACCTTCTGCCAT CTGACTGTC-3'; reverse: 5'-TGCCTGGTTCTGTTGA TAGTGG-3. All primers were purchased from Sangon Biotech Co. Ltd. (Shanghai, China). The $2^{-\Delta \Delta C t}$ method was used for relative quantification of each gene, and $\beta$-actin was used as an internal reference.

\section{Western blot}

Cells were harvested at $90 \%$ confluence by lysis buffer containing phosphatase and protease inhibitor cocktails (Cell Signaling Technology, Danvers, MA, 
USA). For the tissue sample, the sample was ground with liquid nitrogen before homogenizing in lysis buffer. Then, the protein samples were subjected to sodium dodecyl sulfate-polyacrylamide gel electrophoresis. Following that, the proteins were transferred onto a polyvinylidene difluoride membrane (Millipore Corporation, Billerica, MA, USA). After blocking with $5 \%$ skim milk in Tris-buffered saline containing $0.1 \%$ Tween-20 (TBST) at room temperature for $1 \mathrm{~h}$, the membrane was incubated overnight at $4^{\circ} \mathrm{C}$ with the primary antibody as follows: anti-CX3CL1 antibody (1:1000, Abcam, Cambridge, UK), anti-CX3CR1 antibody (1:1000, Abcam, Cambridge, UK), anti-GAPDH antibody (1:5000, Abbkine Inc., CA, USA), and anti-Tubulin antibody (1:5000, Beyotime Biotechnology Corporation, Shanghai, China). Horseradish peroxidase-conjugated goat anti-rabbit or anti-mouse secondary antibody (1:5000, Abbkine Inc., CA, USA) were used to detect the primary antibody. The blots were detected using a chemiluminescence reagent (Millipore Corporation, Billerica, MA, USA).

For the CX3CL1-induced signaling, cells were stimulated with $50 \mathrm{nmol} / \mathrm{L}$ of CX3CL1 (PeproTech, New Jersey, USA) for indicated times $(0,5,15,30,45$, 60 minutes). In blocking experiments, cells were pre-treated with $10 \mathrm{nmol} / \mathrm{L}$ CX3CR1 antagonist (AZD8797, MedChemExpress, NJ, USA) or $1 \mu \mathrm{mol} / \mathrm{L}$ Src or/and FAK inhibitor (Bosutinib and PF-00562271; Selleck, Houston, TX, USA) for 1 hour before stimulation with $50 \mathrm{nmol} / \mathrm{L}$ CX3CL1 for $30 \mathrm{~min}$. The proteins were blotted with monoclonal antibodies to the unphosphorylated and phosphorylated forms of Src (Src, 1:1000; p-Src, 1:1000) and FAK (FAK, 1:1000; p-FAK, 1:500) (Cell Signaling Technology, Danvers, MA, USA). An anti-GAPDH antibody (1:5000, Abbkine Inc., CA, USA) was used to recognizing $\mathrm{GAPDH}$ as the internal reference.

\section{Enzyme-linked immunosorbent assay (ELISA)}

Arterial blood samples were collected before the operation. There were 6 samples from the spinal metastatic breast cancer group and another 6 from the normal control group. The samples were placed at room temperature to clot for 60 minutes before centrifugation for 15 minutes at $1000 \times \mathrm{g}$. Then, serum samples were aliquoted and stored at $-80^{\circ} \mathrm{C}$ until the experiments were performed. The concentration of CX3CL1 in serum was detected using an ELISA kit (R\&D Systems, Minneapolis, MN, USA) according its protocol.

\section{Cell proliferation assay}

Flow cytometry was used to assay cell proliferation. Anti-Ki67 (eBioscience, San Diego, CA,
USA) was used for flow cytometry analysis. Intranuclear staining of Ki67 was performed according to the manufacturer's instructions. Fluorescence-activated cell sorting (FACS) analysis was performed on a CyAn ADP Analyzer (Beckman Coulter, CA, USA), and data were analyzed using FlowJo software (Tree Star, Ashland, OR, USA).

We also used CCK-8 kits to evaluate the effects of CX3CL1 on breast cancer cell proliferation. Cells were grown on a 96-well plate at $1 \times 10^{3}$ cell density per well. After $24 \mathrm{~h}$, the cells were stimulated with 50 nmol/L CX3CL1 in the experimental group. The cells incubated without CX3CL1 were used for the control group. After 1, 2, 3, and 4 days, $10 \mu \mathrm{l}$ Cell Counting Kit-8 (CCK8; Dojindo, Tokyo, Honshu, Japan) reagent was added to the corresponding wells and incubated for another $2 \mathrm{~h}$. Then, the optical density (OD) values at $450 \mathrm{~nm}$ were measured by a spectrophotometer.

\section{Wound-healing assay}

Cells were seeded on a 6 -well plate at $1 \times 10^{5}$ cell density per well and cultured to $90 \%$ confluence. A horizontal wound was created, and then the images were captured. Cells were stimulated with $50 \mathrm{nmol} / \mathrm{L}$ of CX3CL1 for $24 \mathrm{~h}$. The images at $24 \mathrm{~h}$ were also captured, and the migration areas from wound healing were measured and calculated.

\section{In vitro migration and invasion assays}

The 24-well Transwell chamber plates with 8- $\mathrm{mm}$ pores (Corning Co, USA) were used to perform the migration assay, and the chambers coated with Matrigel were used for the invasion assay. A total of $3 \times 10^{4}$ cells for MDA-MB-231 or $5 \times 10^{4}$ cells for MCF-7 in $100 \mu \mathrm{L}$ serum-free medium were added into the upper chamber. The lower chambers were filled with $600 \mu \mathrm{L}$ medium containing $1 \%$ serum that was mixed with or without $50 \mathrm{nmol} / \mathrm{L} \mathrm{CX3CL1}$ and equivalent CX3CL1 blocking antibody (Abcam, Cambridge, UK). For the inhibition assays, the cells in the upper chambers were treated with $1 \mu \mathrm{mol} / \mathrm{L}$ Src or/and FAK inhibitor (Bosutinib and PF-00562271; Selleck, Houston, TX, USA). After incubation at $37^{\circ} \mathrm{C}$ for $24 \mathrm{~h}$, the residual cells in the upper chambers were carefully removed. The cells on the underside of the membranes were fixed with paraformaldehyde and stained with $0.1 \%$ crystal violet. Images were captured at $200 \times$ magnification. Three fields were randomly selected, and the number of cells was counted.

For the invasion assay, the upper chambers were coated with $100 \mu \mathrm{L}$ Matrigel before seeding cells $\left(6 \times 10^{4}\right.$ cells for MDA-MB-231, $10 \times 10^{4}$ cells for MCF-7). The remaining steps were similar to those of the migration assay. 


\section{Statistical analysis}

All data were analyzed with GraphPad Prism software, version 6.0 (GraphPad Software Inc., San Diego, USA) and were expressed as the mean \pm SD. One-way analysis of variance and Student's t-test were used to analyze the differences between groups. $P \leq 0.05$ was considered statistically significant.

\section{Results}

\section{The expression of CX3CR1 and CX3CL1 in the tissue sample}

First, we found that CX3CR1 was highly expressed in tumor tissue by immunohistochemical staining (Supplementary Figure 1). Then, we used RT-PCR and Western blot to detect the expression of CX3CR1 in tumor and para-tumor tissue at RNA and protein levels, respectively. The results of both methods showed that CX3CR1 was significantly more highly expressed in tumor than in para-tumor tissue (Fig. 1A). In terms of CX3CL1, it was a significantly differently expressed between normal spinal cancellous bone and limbs (Fig. 1B).

The concentrations of CX3CL1 in serum samples were detected by ELISA. The serum of healthy people contained a higher level of CX3CL1 than patients with spinal metastases from breast cancer, but the difference was not significant (Fig. 1C).

\section{The expression of CX3CR1 and CX3CL1 in cell lines}

However, CX3CR1 was not expressed at a high level in every breast cancer cell compared with the human mammary epithelial cell line MCF-10A. Interestingly, there was a difference between the RNA and protein levels in MDA-MB-231 cells, which were high in protein levels but low in RNA levels (Fig. 2A-B). We used Flow Cytometry to verify the results of western blot and the results were consistent (Supplementary Figure 3).

Compared with MCF-10A cells, CX3CL1 is highly expressed in MDA-MB-468 cells, followed by MDA-MB-231 cells (Fig 2C-D).

\section{CX3CL1 had no effects on breast cancer cell proliferation}

We first used flow cytometry to evaluate whether CX3CL1 has an impact on MDA-MB-231 cell proliferation. After $48 \mathrm{~h}$ stimulation with $50 \mathrm{nmol} / \mathrm{L}$ CX3CL1, cell proliferation was not promoted
A

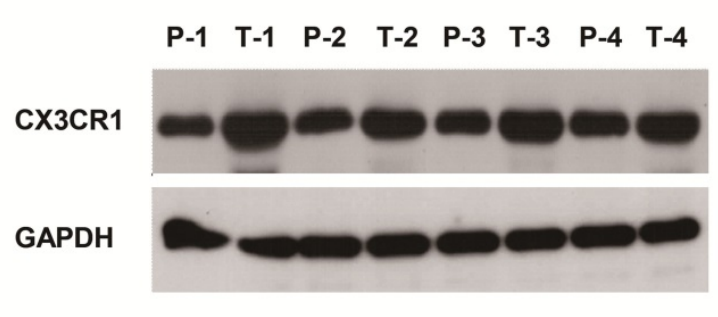

B

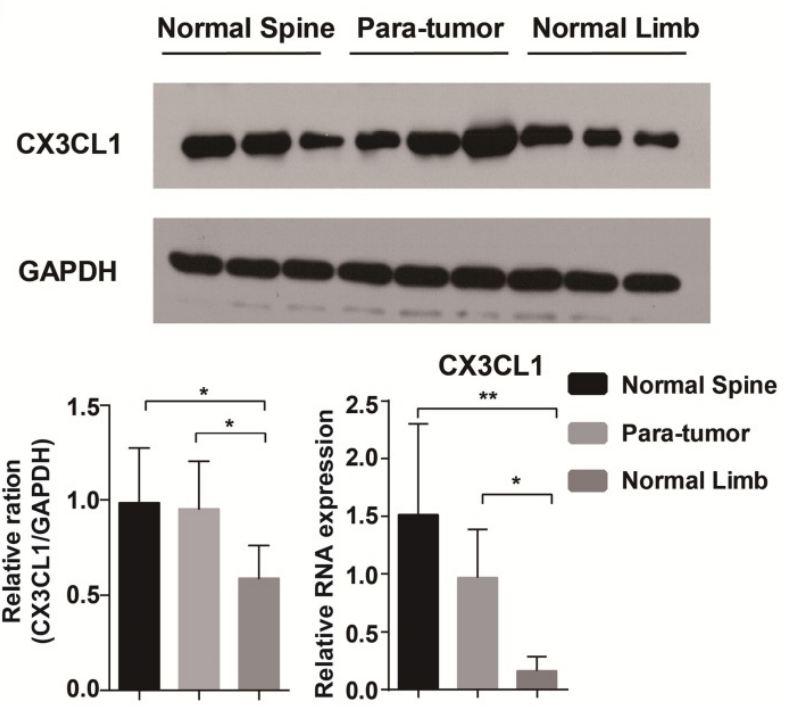

C

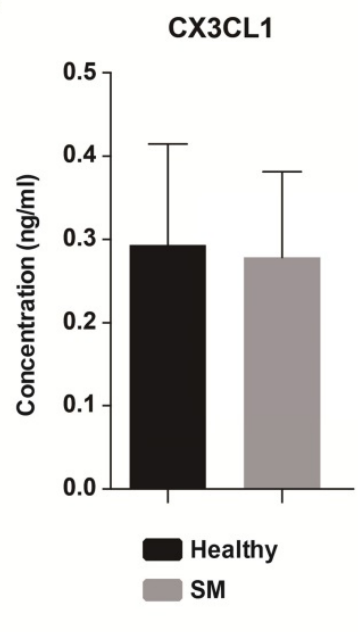

Figure 1. The expression of $C X 3 C R 1$ and $C X 3 C L 1$ in the tissue sample and serum. (A) CX $3 C R 1$ was significantly more expressed in tumor than in para-tumor tissue at RNA and protein levels. P: Para-tumor, T: Tumor. (B) The expression level of CX3CL1 was higher in normal spinal cancellous bone than in limbs. (C) The concentrations of CX3CL1 in serum samples were detected by ELISA. The results were averaged from three independent experiments. SM: Spinal metastasis. *: P<0.05, **P< 0.01 . 
compared with the control group (Fig. 3A). promote cell proliferation over 4 days (Fig. 3B).

Furthermore, the results of the CCK-8 assay revealed that different concentrations of CX3CL1 did not

We verified the result in MCF-7 cells by flow

A

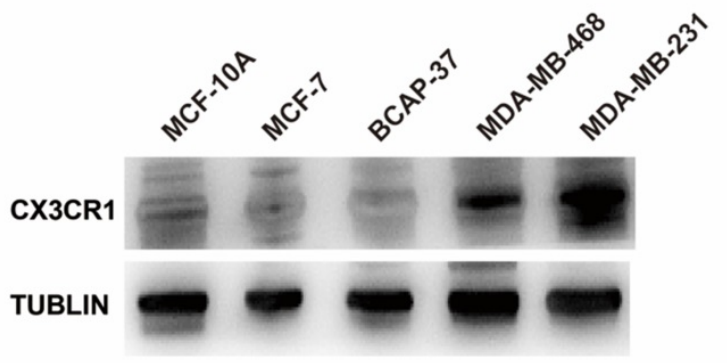

C

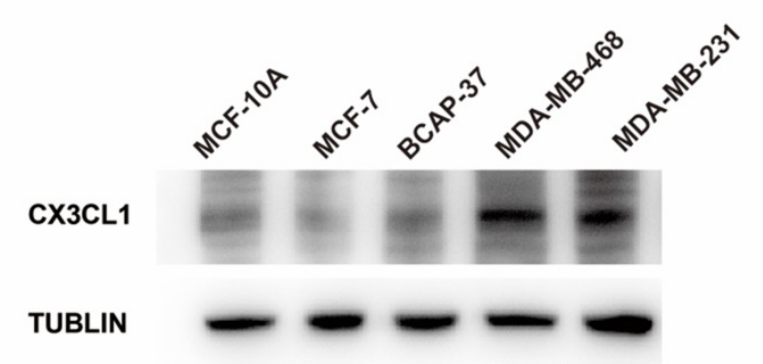

B

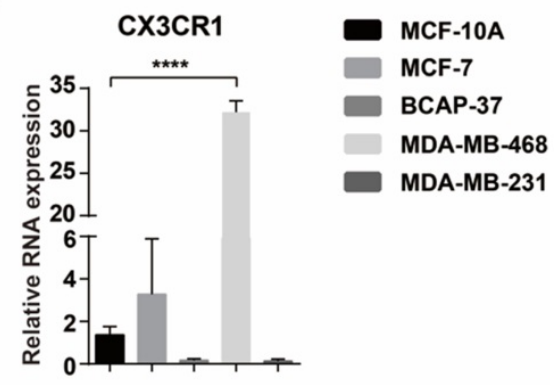

D

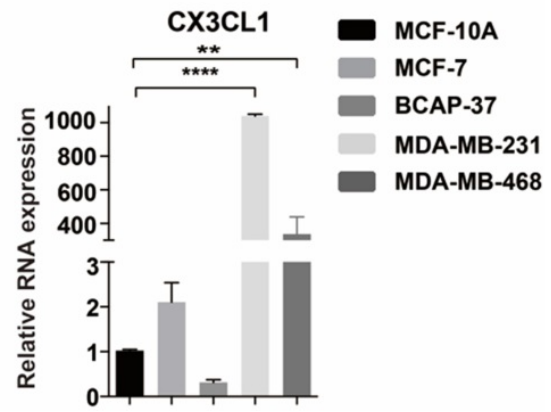

Figure 2. The expression of $C X 3 C R 1$ and $C X 3 C L 1$ in cell lines. (A-B) The expression of CX3CR1 in breast cancer cell lines at protein and RNA levels. (C-D) The expression of $\mathrm{CX} 3 \mathrm{CL} 1$ in breast cancer cell lines at protein and RNA levels. The results were averaged from three independent experiments. $* * \mathrm{P}<0.01$, $* * * * P<0.0001$.

A
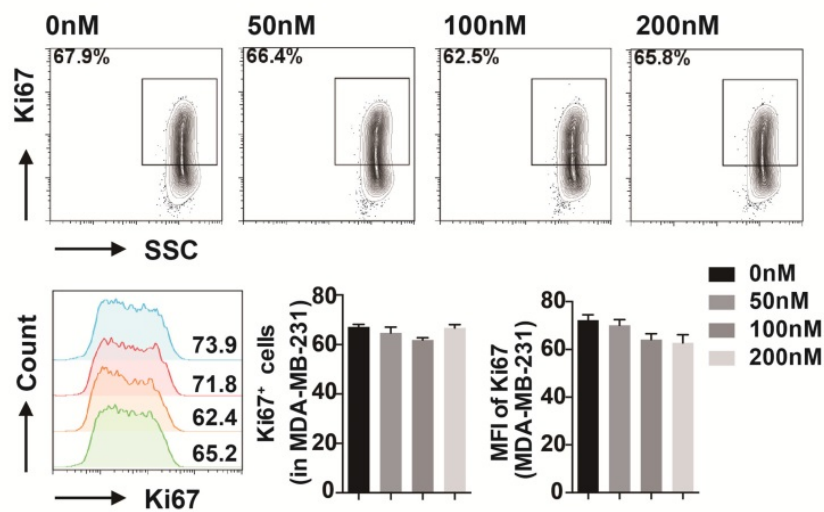

B

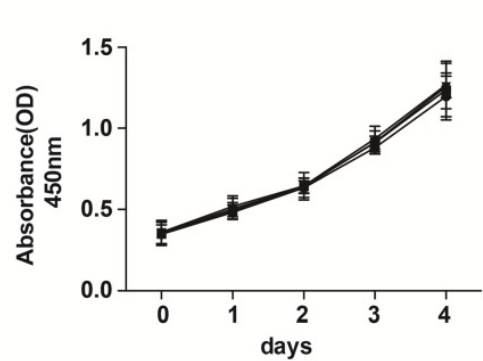

C

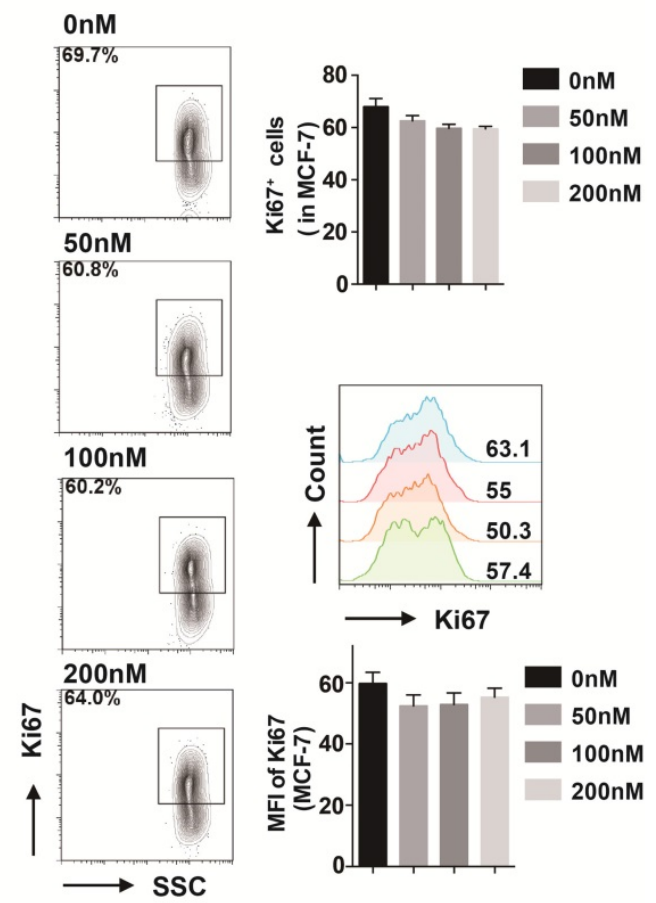

Figure 3. $\mathrm{CX} 3 \mathrm{CL} 1$ had no effects on breast cancer cell proliferation. (A) FACS analysis of Ki67 level in MDA-MB-231 stimulated with 50 nmol/L CX3CL1. (B) Proliferation rate of MDA-MB-231 cells stimulated with different concentrations of CX3CL1 assayed by CCK-8. (C) FACS analysis of Ki67 level in MCF-7 cells stimulated with different concentrations of $\mathrm{CX} 3 \mathrm{CL} 1$. The results were averaged from three independent experiments. 


\section{A}
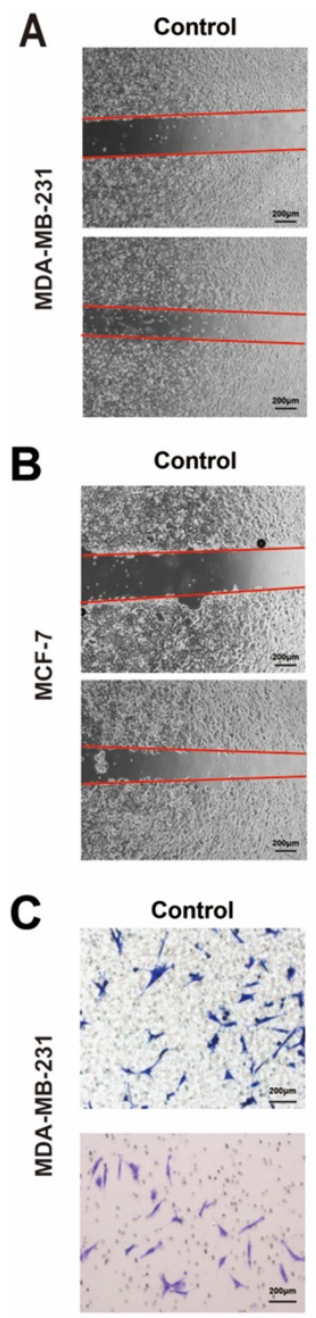

D

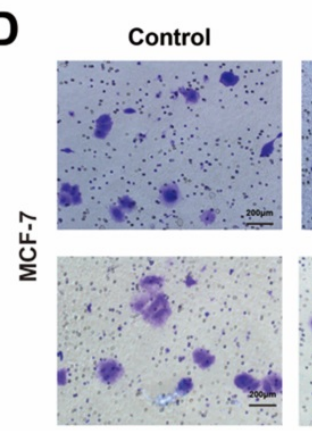

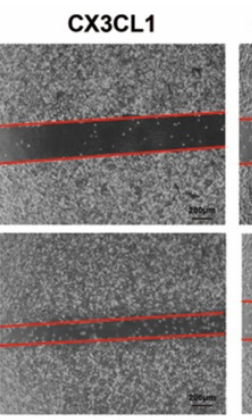

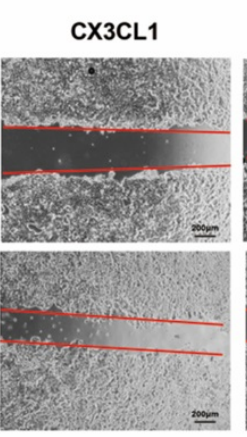

CX3CL1
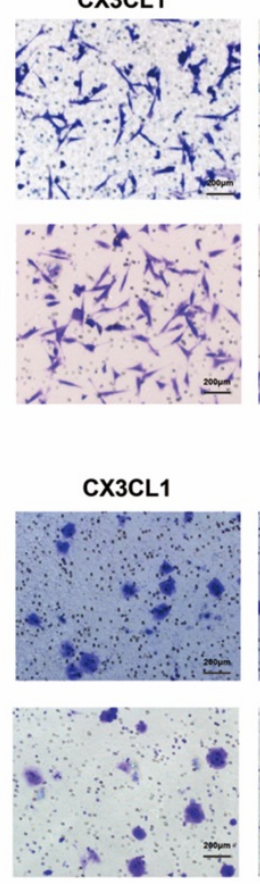

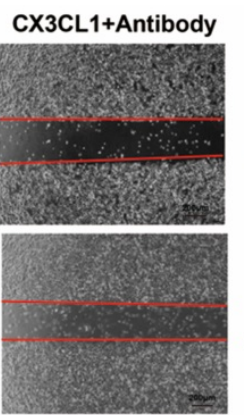

CX3CL1+Antibody
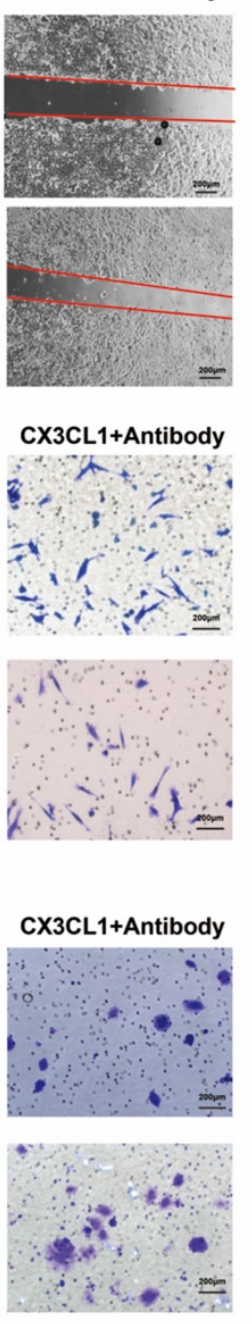

oh

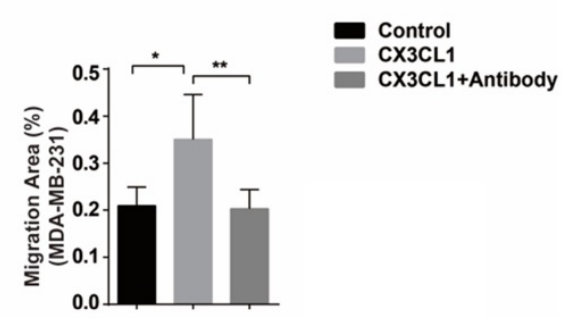

on
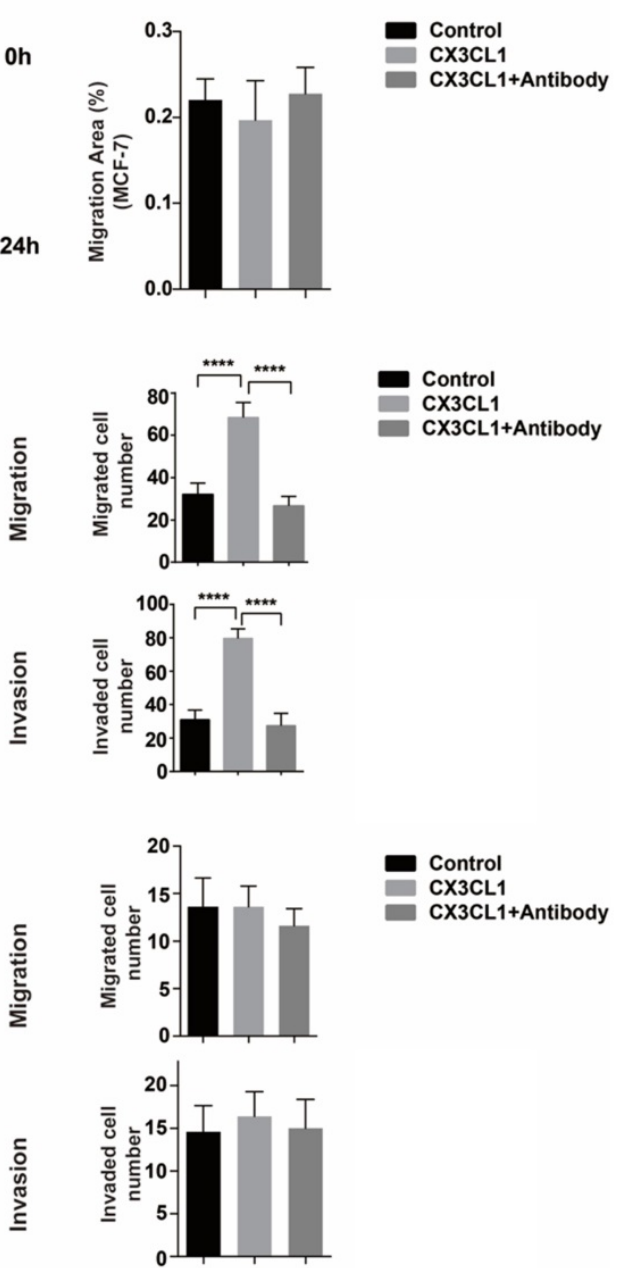

Figure 4. $C \times 3 C L 1$ promotes the migration and invasion abilities of $C X 3 C R 1$-expressing cells. (A) Wound healing assays of MDA-MB-231 cells and MCF-7 cells treated without (control) or with $50 \mathrm{nmol} / \mathrm{L} \mathrm{CX3CL1}$ and with neutralizing antibody. (B) The migration and invasion assays of MDA-MB-231 cells treated without (control) or with 50 nmol/L $\mathrm{CX} 3 \mathrm{CL} 1$ and with neutralizing antibody. Scale bar $=200 \mu \mathrm{m}$. (C) The migration and invasion assays of MCF-7 cells treated without (control) or with $50 \mathrm{nmol} / \mathrm{L} \mathrm{CX} 3 \mathrm{CL} 1$ and with neutralizing antibody. Scale bar $=200 \mu \mathrm{m}$. The results were averaged from three independent experiments. $* \mathrm{P}<0.05, * * \mathrm{P}<0.01, * * * \mathrm{P}<0.001, * * * * \mathrm{P}<0.0001$.

CX3CLI promotes the migration and invasion abilities of CX3CR1-expressing cells

Wound-healing and migration assays showed that MDA-MB-231 presented with superior migration ability when induced by CX3CL1 at a concentration of $50 \mathrm{nmol} / \mathrm{L}$ compared with the control group (Fig. $4 \mathrm{~A}$ and $4 \mathrm{C}$ top). However, this phenomenon can be blocked by CX3CL1-neutralizing antibody. Meanwhile, in terms of MCF-7 cells, which expressed minimal level of CX3CR1, CX3CL1 did not function (Fig. $4 \mathrm{~B}$ and $4 \mathrm{D}$ top).

The invasion ability was investigated using Matrigel. Similar to the migration assay, CX3CL1 significantly increased the invasion ability of MDA-MB-231 and was also blocked by neutralizing antibody (Fig. 4C bottom). Similarly, it did not function in MCF-7 cells (Fig. 4D bottom). 
A
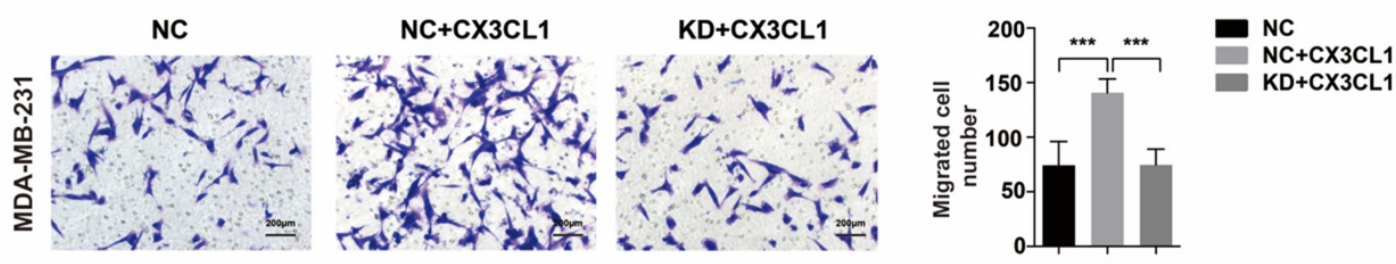

B
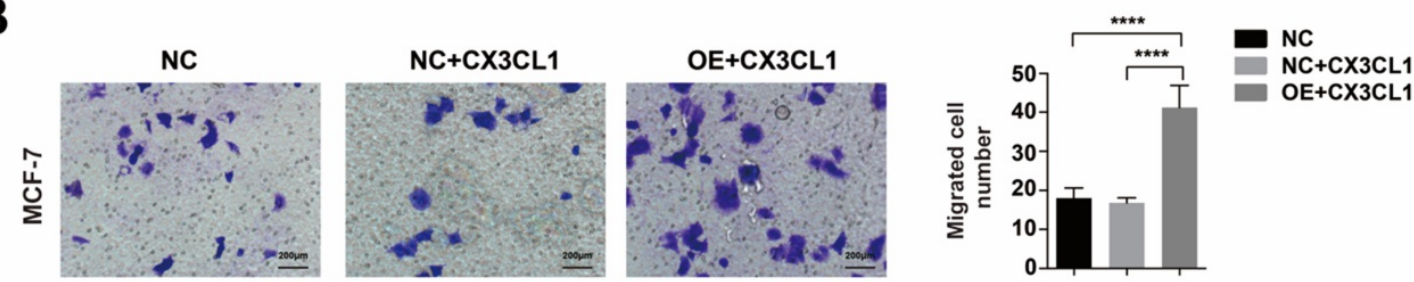

Figure 5. Migration assays of MDA-MB-231 CX3CR1-knockdown (KD) cells and MCF-7 CX3CR1-overexpressing (OE) cells. (A) Migration assay of MDA-MB-231 (KD) cells. (B) Migration assay of MCF-7(OE) cells. The results were averaged from three independent experiments. NC: Negative control, KD: knockdown, OE: Overexpress. ***P< 0.001 , $* * * * \mathrm{P}<0.0001$.

Additionally, when down-regulating CX3CR1 expression in MDA-MB-231 cells (Supplementary Figure 2A-B), CX3CL1 did not stimulate cell migration (Fig 5A). And when overexpressing CX3CR1 expression in MCF-7 cells (Supplementary Figure 2C-D), the number of migrated cells was significantly increased after stimulated by CX3CL1 (Fig 5B).

\section{CX3CL1 induced chemotaxis of tumor cells via the Src/FAK signaling pathway in CX3CR1-expressing cells}

To demonstrate that CX3CL1 induced chemotaxis of tumor cells via the Src/FAK signaling pathway, we detected the phosphorylation of Src and FAK in cells stimulated with CX3CL1 for indicated times. The results showed that in MDA-MB-231 cells, the Src phosphorylation started 5 minutes after stimulation, peaked at 15 minutes, and continued through 60 minutes; FAK phosphorylation started at 5 minutes, peaked at 30 minutes, and then returned to baseline levels gradually (Fig. 6A). However, compared with $0 \mathrm{~min}$, there was no significant increase in the phosphorylation levels of Src and FAK, and there was no significant correlation between phosphorylation levels of Src and FAK and time in MCF-7 cells (Fig. 6B).

When MDA-MB-231 cells were pretreated with the CX3CR1 antagonist AZD8797, the phosphorylation levels of $\mathrm{Src}$ and FAK were significantly decreased (Fig. 6C). Additionally, Bosutinib and PF-00562271 can suppress the phosphorylation level of Src and FAK induced by CX3CL1, especially using a combination of the two inhibitors (Fig. 6D). On the other hand, the results were further confirmed by migration assay. The migration index enhanced by CX3CL1 was dramatically reduced using Bosutinib and PF-00562271 (Fig. 6E).

\section{Discussion}

CX3CL1 was detected in 1997 as a unique member of the CX3C family. Unlike other chemokines, CX3CL1 is a transmembrane glycoprotein containing 373 amino acids and a chemokine domain on the top of the mucin-like stalk [9]. This molecule exists in two forms: the membrane-attached form and the shed form. The soluble fragment of CX3CL1 is cleaved from the membrane-attached CX3CL1 under normal conditions. Previous studies showed that the CX3CL1/CX3CR1 axis regulated interaction between neurons and glial cells in the central nervous system and was implicated in glioma $[11,16]$. As the research further developed, more and more studies have shown that CX3CR1 is expressed on multiple malignant tumor cells, and the CX3CL1/CX3CR1 axis was associated with tumor metastasis, including prostate cancer, renal cancer, gastric cancer, and breast cancer $[13,14,17,18]$. Although several reports have demonstrated that CX3CL1 is involved in the metastatic process of a variety of malignant tumors, there have been few studies on the role of CX3CL1 in breast cancer metastasizing to the spine. The present study indicated that CX3CL1 may play a particular role in the process of breast cancer metastasizing to the spine. Our findings suggested that CX3CL1 could promote the chemotaxis ability of breast cancer cells by interacting with CX3CR1 via the Src/FAK signaling pathway. 

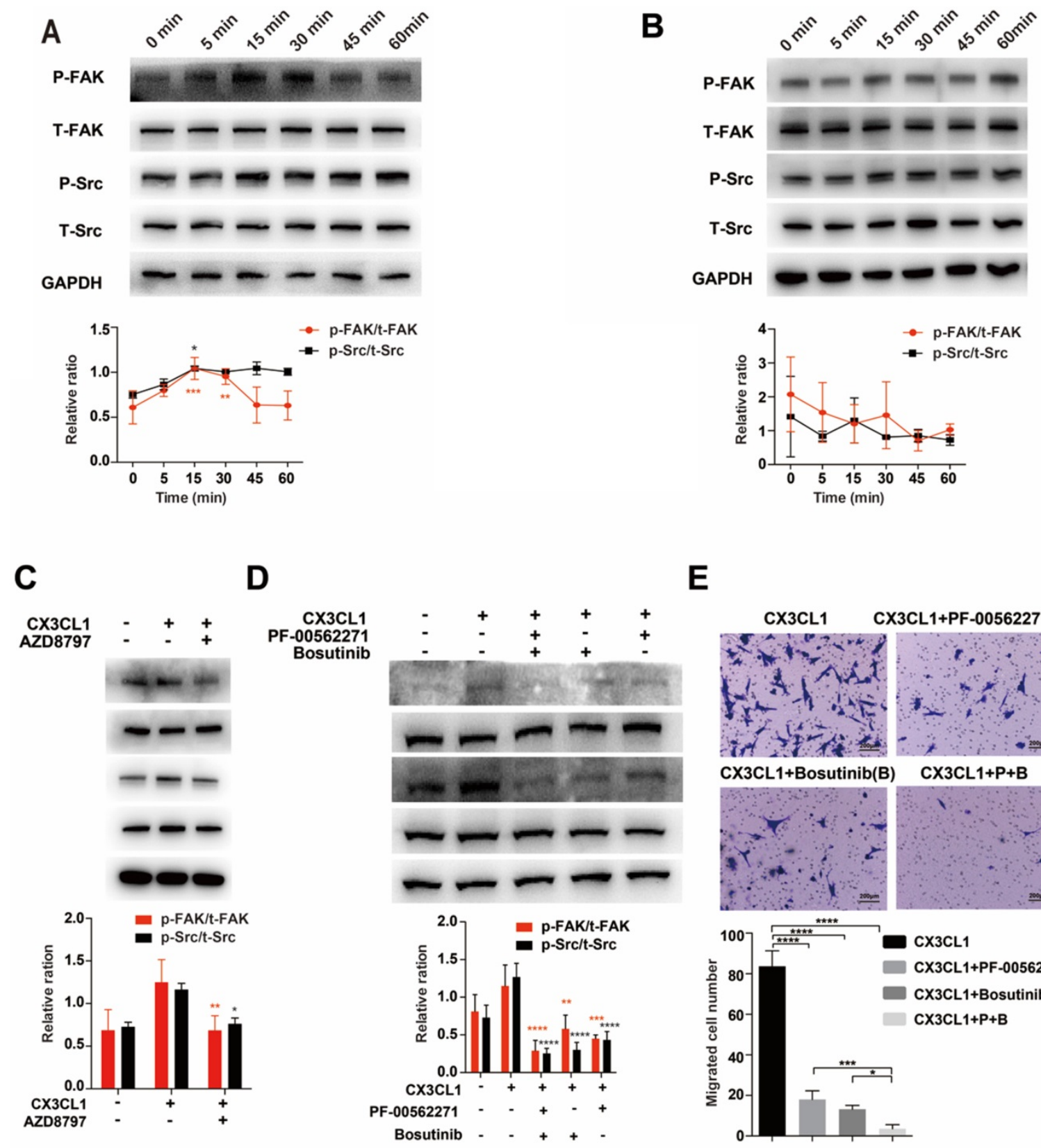

$\mathbf{E}$

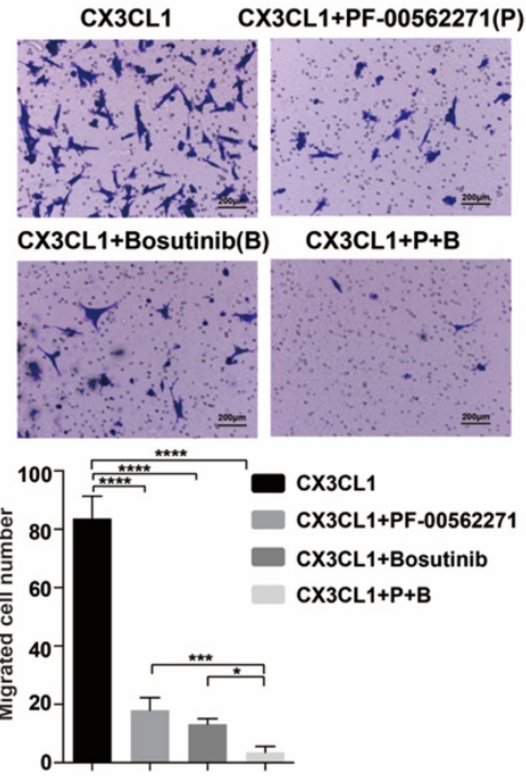

Figure 6. CX3CLI induced chemotaxis of tumor cells via the Src/FAK signaling pathway in CX3CR1-expressing cells. (A) Phosphorylation levels of Src and FAK of MDA-MB-231 cells after stimulated by CX3CL1 for indicated time 0 min as control. (B) Phosphorylation levels of Src and FAK of MCF-7 cells after stimulated by CX3CL1 for indicated time. 0 min as control. (C) MDA-MB-231 cells were pretreated with the CX3CR 1 antagonist AZD8797, and stimulated by CX3CL1 for 30 mins, the phosphorylation levels of Src and FAK were detected. CX3CL1-only group as control. (D) MDA-MB-231 cells were pretreated with the inhibitors of Src and FAK pathways, Bosutinib and PF-00562271, and stimulated by CX3CL1 for 30 mins, the phosphorylation levels of Src and FAK were detected. CX3CL1-only group as control. (E) The migration index enhanced by $C X 3 C L 1$ was dramatic reduced using Bosutinib and PF-00562271. CX3CL1-only group as control. Scale bar $=200 \mu \mathrm{m}$. The experiments were repeated three times. $* \mathrm{P}<0.05$, ${ }^{*} \mathrm{P}<0.01$, $* * * \mathrm{P}<0.001$, $* * * * \mathrm{P}<0.0001$.

We first investigated the expression of CX3CR1 in specimens from spinal metastatic breast cancer and para-tumor tissues, and the results of western blot, real-time PCR and immunohistochemistry all indicated that the expression level of CX3CR1 was higher in tumor tissue than in para-tumor tissue. Moreover, the expression level of CX3CL1 was higher in normal spinal cancellous bone than in limbs, which may indicate that the spine is a prior target for circulating breast cancer cells expressing CX3CR1 and explain why breast cancer is more likely to metastasize to the spine rather than limbs in clinical settings $[7,8]$. However, these results have not yet been reported in the literature.

We also investigated the expression of CX3CR1 in different breast cancer cells at the RNA and protein levels. The results showed that the expression of CX3CR1 was different in each breast cancer cell at the RNA and protein levels. However, there was a difference between RNA and protein levels in MDA-MB-231 cells. This finding may be attributed to posttranscription or posttranslational regulation 
mechanisms, which means the amount of protein synthesis is not consistent with the amount of RNA in some cases [19]. Because MDA-MB-231 cells have a high expression of CX3CR1 at the protein level and are known as strongly bone-metastatic [20, 21], we selected them for the further study. Meanwhile, MCF-7 cells, which expressed minimal levels of CX3CR1, were selected as the comparison cells.

The effects of CX3CL1 on cell proliferation were detected by flow cytometry and CCK-8 assay. We found that CX3CL1 had no effects on proliferation of the two kinds of breast cancer cells. In agreement with our results, Yao $\mathrm{X}$ et al. found the same pattern in renal cancer cells [14]. However, Tardaguila et al. reported that CX3CL1 induced breast cancer cell T47D proliferation via transactivation of the EGF pathway [13]. This difference may be explained by the fact that even in the same tumor, different subtypes have different responses to the same stimulating factor, which is called tumor heterogeneity.

According to our results from cell proliferation, wound healing, in vitro migration and invasion assays, CX3CL1 promoted the migration and invasion abilities of MDA-MB-231 cells instead of enhancing growth properties. However, it did not function in MCF-7 cells, which have low expression of CX3CR1. These findings demonstrated that the chemotaxis process induced by CX3CL1 is dependent on the expression of a chemokine-matched receptor on cancer cells. Meanwhile, the neutralizing antibody of CX3CL1 can interfere with the migration process induced by CX3CL1. Coincidentally, Yao X et al. confirmed that CX3CL1 induced migration of renal cancer cells with CX3CR1 membrane expression [14]. In another study, Shulby SA et al. demonstrated that CX3CL1 was also involved in migration of human prostate cancer cells [17]. Therefore, we concluded that CX3CL1 may play an important role in the migration of various cancer cells.

To study the molecular mechanisms of CX3CL1 activation in breast cancer cells, MDA-MB-231 cells were stimulated with CX3CL1 for indicated times. Based on our observations, time-dependent phosphorylation of Src (Tyr 416) and FAK (Tyr576/577) was detected. Src is a non-receptor protein, tyrosine kinase, induced by many classes of cellular signal molecules and plays an important role in regulating numerous processes, including cell growth, differentiation, adhesion and the migration signaling pathway $[22,23]$. Src activity is mediated by tyrosine phosphorylation at two sites, Tyr416 and Tyr527, which have opposite effects. When phosphorylation occurs at Tyr416, the enzyme activity is upregulated, which was observed in our study. However, phosphorylation at Tyr527 renders the enzyme less active [22]. It has been well studied that Src play important role in cell proliferation [24]. However, our study found that the activation of the Src pathway did not affect cell proliferation. Tsai et al reported that in MDA-MB-231 cells, cell proliferation had not been affected when Src signaling pathway was inhibited [25]. Two other researches even found that activation of the Src pathway could inhibit cell proliferation in colon cancer cells and endothelial cells $[26,27]$. Therefore, we believed that the Src signaling pathway may exhibit a specific role in specific situations. Focal adhesion kinase (FAK) is another widely expressed non-receptor protein tyrosine kinase involved in regulating several biological processes, including cell spreading, proliferation, and migration [28, 29]. The activation of FAK requires auto-phosphorylation at Tyr397 via integrin clustering. Meanwhile, Tyr397 is also a high-affinity binding site for Src. Recruitment of Src family kinases then phosphorylate Tyr576/577 in the catalytic domain [30, 31]. Combined with our results, we suggest that CX3CL1 phosphorylates Src at Tyr416 via interaction with CX3CR1, and then phosphorylated Src phosphorylates FAK at Tyr576/577 by binding to FAK at Tyr397, thereby promoting cell migration. Additionally, CX3CR1 antagonist AZD8797 could inhibit the phosphorylation levels of Src and FAK. It is further proved that CX3CL1 needs to interact with CX3CR1 to function. Furthermore, this process also could be blocked by inhibitors of the Src and FAK pathway, Bosutinib and PF-00562271, respectively, especially using a combination of both inhibitors. Researchers have reported that these two inhibitors could block cell movement in some kinds of cancer as well, which is consistent with our results [32, 33]. Thus, Bosutinib and PF-00562271 may translate to clinical application for blocking cancer metastasis, but more experiments are needed to verify these findings.

\section{Conclusion}

As far as we know, this is the first study suggesting that the CX3CL1/CX3CR1 axis is associated with the process of breast cancer metastasizing to the spine. In conclusion, CX3CL1 in spinal cancellous bone attracts CX3CR1-expressing tumor cells to the spine and enhances their migration and invasion abilities through the Src/FAK signaling pathway. Our results also suggest that Bosutinib and PF-00562271 have anti-metastasis potential.

\section{Supplementary Material}

Supplementary materials and methods, figures. http://www.jcancer.org/v09p3603s1.pdf 


\section{Acknowledgements}

This work was sponsored by Natural Science Foundation of China (grant number 81572629 and 81772855).

\section{Competing Interests}

The authors have declared that no competing interest exists.

\section{References}

[1] Schulman KL and Kohles J. Economic burden of metastatic bone disease in the U.S[J]. Cancer,2007, 109(11): 2334-42.

[2] Perrin RG and Laxton AW. Metastatic spine disease: epidemiology, pathophysiology, and evaluation of patients[J]. Neurosurg Clin N Am,2004, 15(4): 365-73.

[3] Katanoda $\mathrm{K}$ and Matsuda T. Five-year relative survival rate of breast cancer in the USA, Europe and Japan[J]. Jpn J Clin Oncol,2014, 44(6): 611.

[4] Forouzanfar MH, Foreman KJ, Delossantos AM, et al. Breast and cervical cancer in 187 countries between 1980 and 2010: a systematic analysis[J]. Lancet,2011, 378(9801): 1461-84.

[5] Ferlay J, Shin HR, Bray F, et al. Estimates of worldwide burden of cancer in 2008: GLOBOCAN 2008[J]. Int J Cancer,2010, 127(12): 2893-917.

[6] Coleman RE and Rubens RD. The clinical course of bone metastases from breast cancer[J]. Br J Cancer,1987, 55(1): 61-6.

[7] Briasoulis E, Karavasilis V, Kostadima L, et al. Metastatic breast carcinoma confined to bone: portrait of a clinical entity[J]. Cancer,2004, 101(7): 1524-8.

[8] Sarabia-Estrada R, Ruiz-Valls A, Guerrero-Cazares H, et al. Metastatic human breast cancer to the spine produces mechanical hyperalgesia and gait deficits in rodents[J]. Spine J,2017, 17(9): 1325-1334.

[9] Bazan JF, Bacon KB, Hardiman G, et al. A new class of membrane-bound chemokine with a CX3C motif[J]. Nature,1997, 385(6617): 640-4.

[10] Kim KW, Vallon-Eberhard A, Zigmond E, et al. In vivo structure/function and expression analysis of the CX3C chemokine fractalkine[J]. Blood,2011, 118(22): e156-67.

[11] Marchesi F, Locatelli M, Solinas G, et al. Role of CX3CR1/CX3CL1 axis in primary and secondary involvement of the nervous system by cancer[J]. J Neuroimmunol,2010, 224(1-2): 39-44.

[12] Ryu J, Lee CW, Hong KH, et al. Activation of fractalkine/CX3CR1 by vascular endothelial cells induces angiogenesis through VEGF-A/KDR and reverses hindlimb ischaemia[J]. Cardiovasc Res,2008, 78(2): 333-40.

[13] Tardaguila M, Mira E, Garcia-Cabezas MA, et al. CX3CL1 promotes breast cancer via transactivation of the EGF pathway[J]. Cancer Res,2013, 73(14): 4461-73.

[14] Yao X, Oi L, Chen X, et al. Expression of CX3CR1 associates with cellular migration, metastasis, and prognosis in human clear cell renal cell carcinoma[J]. Urol Oncol,2014, 32(2): 162-70.

[15] Liu W, Bian C, Liang Y, et al. CX3CL1: a potential chemokine widely involved in the process spinal metastases[J]. Oncotarget,2017, 8(9): 15213-15219.

[16] Feng X, Szulzewsky F, Yerevanian A, et al. Loss of CX3CR1 increases accumulation of inflammatory monocytes and promotes gliomagenesis[J]. Oncotarget,2015, 6(17): 15077-94.

[17] Shulby SA, Dolloff NG, Stearns ME, et al. CX3CR1-fractalkine expression regulates cellular mechanisms involved in adhesion, migration, and survival of human prostate cancer cells[J]. Cancer Res,2004, 64(14): 4693-8.

[18] Wei LM, Cao S, Yu WD, et al. Overexpression of CX3CR1 is associated with cellular metastasis, proliferation and survival in gastric cancer[J]. Oncol Rep, 2015, 33(2): 615-24.

[19] Ideker T, Thorsson V, Ranish JA, et al. Integrated genomic and proteomic analyses of a systematically perturbed metabolic network[J]. Science,2001, 292(5518): 929-34.

[20] Jamieson-Gladney WL, Zhang $\mathrm{Y}$, Fong AM, et al. The chemokine receptor CX(3)CR1 is directly involved in the arrest of breast cancer cells to the skeleton[J]. Breast Cancer Res,2011, 13(5): R91.

[21] Kang Y, Siegel PM, Shu W, et al. A multigenic program mediating breast cancer metastasis to bone[J]. Cancer Cell,2003, 3(6): 537-49.

[22] Thomas SM and Brugge JS. Cellular functions regulated by Src family kinases[J]. Annu Rev Cell Dev Biol,1997, 13: 513-609.

[23] Parsons SJ and Parsons JT. Src family kinases, key regulators of signal transduction[J]. Oncogene,2004, 23(48): 7906-9.

[24] Roskoski R. Src protein-tyrosine kinase structure and regulation[J]. Biochem. Biophys. Res. Commun.,2004, 324(4): 1155-64.

[25] Tsai P, Chu C, Chiu C, et al. Inhibition of Src activation with cardiotoxin III blocks migration and invasion of MDA-MB-231 cells[J]. Toxicon,2013, 74: 56-67.

[26] Kuo CT, Chang C, and Lee WS. Folic acid inhibits COLO-205 colon cancer cell proliferation through activating the FRalpha/c-SRC/ERK1/2/NFkappaB/ TP53 pathway: in vitro and in vivo studies[J]. Sci Rep,2015, 5: 11187.
[27] Lin S, Lee W, Su Y, et al. Folic acid inhibits endothelial cell proliferation through activating the cSrc/ERK $2 / \mathrm{NF}-\mathrm{KB} / \mathrm{p} 53$ pathway mediated by folic acid receptor[J]. Angiogenesis,2012, 15(4): 671-83.

[28] Parsons JT, Martin KH, Slack JK, et al. Focal adhesion kinase: a regulator of focal adhesion dynamics and cell movement[J]. Oncogene,2000, 19(49): 5606-13.

[29] Cox BD, Natarajan M, Stettner MR, et al. New concepts regarding focal adhesion kinase promotion of cell migration and proliferation[J]. J Cell Biochem,2006, 99(1): 35-52.

[30] Schaller MD, Hildebrand JD, Shannon JD, et al. Autophosphorylation of the focal adhesion kinase, pp125FAK, directs SH2-dependent binding of pp60src[J]. Mol Cell Biol,1994, 14(3): 1680-8.

[31] Hanks SK, Ryzhova L, Shin NY, et al. Focal adhesion kinase signaling activities and their implications in the control of cell survival and motility[J]. Front Biosci,2003, 8: d982-96.

[32] Vultur A, Buettner R, Kowolik C, et al. SKI-606 (bosutinib), a novel Src kinase inhibitor, suppresses migration and invasion of human breast cancer cells[J]. Mol Cancer Ther,2008, 7(5): 1185-94.

[33] Wang Z, Wang Z, Li G, et al. CXCL1 from tumor-associated lymphatic endothelial cells drives gastric cancer cell into lymphatic system via activating integrin beta1/FAK/AKT signaling[J]. Cancer Lett,2017, 385: 28-38. 